\title{
Establishment of the coarse grained parameters for epoxy-copper interfacial separation
}

\author{
Cell K. Y. Wong, ${ }^{1,2}$ Stanley Y. Y. Leung, ${ }^{1}$ Rene H. Poelma, ${ }^{2}$ Kaspar M. B. Jansen, ${ }^{3}$ \\ Cadmus C. A. Yuan, ${ }^{2,4}$ Willem D. van Driel, ${ }^{2,5}$ and Guoqi Zhang ${ }^{1,2,5}$ \\ ${ }^{1}$ Faculty $3 m E$, Department PME, Delft University of Technology, Delft, The Netherlands \\ ${ }^{2}$ DIMES, Delft University of Technology, Delft, The Netherlands \\ ${ }^{3}$ Industrial Design Engineering, Product Engineering Section, Delft University of Technology, \\ Delft, The Netherlands \\ ${ }^{4}$ TNO IenT, Materials Technology, De Rondom 1, Eindhoven, The Netherlands \\ ${ }^{5}$ Philips Lighting, Mathildelaan 1, Eindhoven, The Netherlands
}

(Received 7 November 2011; accepted 7 April 2012; published online 9 May 2012)

\begin{abstract}
Atomistic coarse grained parameters were calculated from a non-equilibrium molecular dynamics simulation of the separation of an epoxy-copper interface. The methodology to determine the interaction energy and the equilibrium distance between the interfacial materials at a minimum energy is established. The traction-displacement relations of the separation under the influence of time taken for atomic interaction, displacement step, and molecular size have been studied. The study illustrates that the control of the time step in the molecular dynamics models is important to ensure a proper separation simulation. The result shows close matching with the thermodynamics work of adhesion. An analytical scheme to determine the coarse grained parameters from the relations is discussed. The proposed methodology contributes to the interpretation of interfacial adhesion beyond the continuum framework. (c) 2012 American Institute of Physics. [http://dx.doi.org/10.1063/1.4712060]
\end{abstract}

\section{INTRODUCTION}

Polymer-metal interfacial adhesion is one of the most important phenomena in the field of science and technology. ${ }^{1,2}$ The adhesion technology has been widely applied, both structurally and functionally, in industries such as electronic packaging and advanced composite. Prediction of the interfacial properties of the joint materials during their service life has been a concern of the product reliability. ${ }^{3,4}$ The conventional method, which involves continuum fracture mechanics, requires the analysis of stresses well behind the crack tip processing zone. As the feature size of the electronic products or the filler size of the composite approaches nano-scale, the processing zone size becomes dominant within the analyzed structure. Thus, the applicability of fracture mechanics becomes problematic.

Recent development in atomistic modeling enables the study of the structural, thermal, dynamics and mechanical properties of materials in nanoscale. ${ }^{5-9}$ While the atomistic simulations of fracture behavior of metal or crystalline solids are quite successful, ${ }^{10-12}$ the modeling of polymer in atomistic detail is subjected to time and length scales limitations. Coarse grained approaches, which use pseudo-atoms (beads) to represent groups of atoms so as to reduce the number of particles taken part in the interaction, help to overcome these limitations. ${ }^{13-17}$

One of the major concern in the coarse grained model is the parameterization of the forcefield. In the case of interfacial separation, parameters that describe the energy required for the separation process is considered. ${ }^{18-21}$ The two parameters that describe the interfacial separation phenomenon are (1) the interaction energy required for the separation, $\gamma$, and
(2) the equilibrium distance between the interfacial materials at a minimum energy, $r_{e}$. The parameterization are determined empirically by matching the physical quantity calculated from the coarse grained model to appropriate experimental data ${ }^{22}$ or estimating the physical parameters from atomistic simulations. ${ }^{23}$ Despite the importance in obtaining the parameters, characterization of the interface phenomenon is limited to mesoscopic scale due to the experimental difficulties. ${ }^{20}$ The parameterization based on the matching of nano-scale experimental data is therefore obstructed.

Recent effort has been concentrated in deducing the coarse grained parameters through atomistic models. Semoto et $a .^{20}$ studied the adhesion between a $\gamma$-alumina surface and epoxy with density function theory (DFT) calculations. Force-distance relations, which describes the force required to separate the interface, has been derived indirectly from the single-point energy of the system upon displacement of the epoxy. Iwamoto ${ }^{21}$ investigated the separation of an epoxy-copper oxide system with the molecular dynamics (MD) approach, the maximum change in potential energy upon separation was taken as the interaction energy and the average separation distance was used to estimate the equilibrium distance. Yang et $a l^{24}$ considered the stressdisplacement relations for the separation of an intimate contacted epoxy-copper system with a molecular model. They applied molecular statics (MS) to calculate the reaction force of the copper atoms at different separation distances to the epoxy and to obtain the interaction energy. Nevertheless, in omitting the temperature effect (energy minimization at zero Kelvin), the model neglects time dependent molecular motion. ${ }^{25}$ The previous literature shows no consideration of 
the influencing parameters in the atomistic model, which alters the separation process, the coarse grained parameters deduced from these methods are doubtful.

The interfacial separation is a dynamic process, which involves the interaction of atoms in the polymer and metal layer. The shape of a traction (reaction force)-displacement relation is governed by the atomic interactions at the interface. The governing factors are (i) time taken for the atoms to interact; (ii) displacing distance of one of the layer in each separation step; and (iii) the molecular size of the polymer involved in the separation (model size). Here, in considering the three influencing parameters (as described in Fig. 2), we deduce the force-displacement relations of the separation of epoxy-copper interface with molecular dynamics approach. The relations are then used to derive the coarse grained parameters needed for mesoscale model development.

\section{NON-EQUILIBRIUM MOLECULAR DYNAMICS MODEL FOR EPOXY-COPPER INTERFACE}

The interfacial model consists of two layers: copper and epoxy. The copper layer was created with a crystalline copper structure cleaved along the (001) plane. The epoxy layer, which was comprised of tri- and tetra- epoxy novolac functionalized resins and hydroxyl hardener monomers, was built in an amorphous unit cell with a density set as $1.2 \mathrm{~g} / \mathrm{cm}^{3}$ based on commercialized epoxy compounds. An interfacial model was then constructed by combining the two layers. The size of the interfacial model unless specific was $28 \AA \times 56 \AA$ which contained 1538 copper atoms, 8 trifunctional epoxy monomers, 12 tetrafunctional epoxy monomers, and 36 hydroxyl hardeners.

In order to construct a crosslinked structure of the epoxy layer, crosslinking of the epoxy and hardener monomers was simulated according to the procedure reported previously. $^{24,26}$ A three-dimensional polymeric structure was built. The models were assigned with a non-periodic boundary conditions, in order to avoid the over constraint induced at the boundary. ${ }^{27}$ The interfacial structure was then relaxed to remove the constraints induced during model construction according to the procedures listed in Appendix Table IV. The bi-materials atomistic model assumes no chemical bond is linking between the epoxy and the copper. The separation
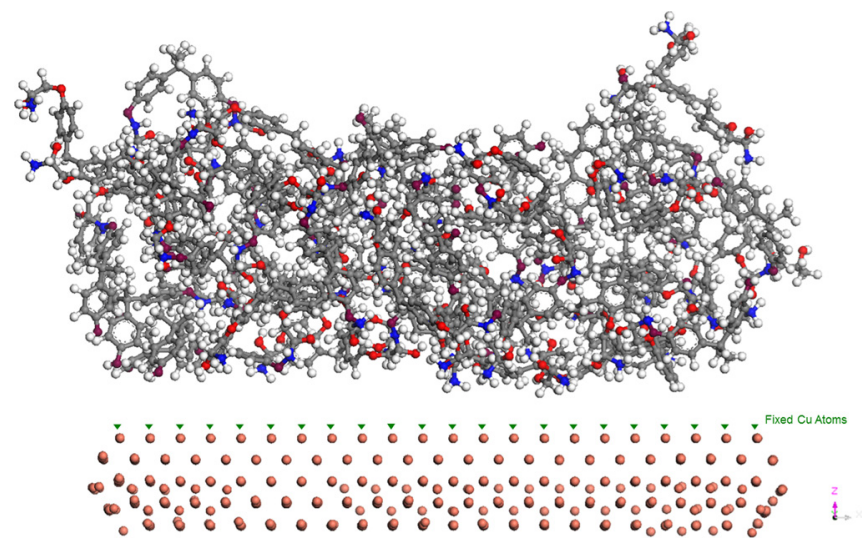

FIG. 1. Initial configuration of the interfacial model in non-periodic boundary conditions. involves only physical interaction, which results in an atomically clean separation.

The forcefield simulation was performed with the Forcite module of MATERIAL STUDIO 5.0 software (Accelrys, Inc). ${ }^{28}$ To describe the interatomic interactions of the interface, the condensed-phase optimized molecular potentials for atomistic simulation studies (COMPASS) forcefield have been utilized with a cutoff distance set at $9.5 \AA$. Due to the fact that parameterization of the COMPASS forcefield has been conducted by fitting against a wide range of experimental data for organic and inorganic compounds, it has been commonly applied in studying the polymer-metal interfaces. ${ }^{29-31}$

The initial configuration of the model is illustrated in Fig. 1. All atoms, except those in the topmost layer of the copper atoms, were set free to move in $\mathrm{x}, \mathrm{y}$, and $\mathrm{z}$ direction. The copper was defined to be the displacement layer. An incremental displacement along the negative z-direction was given in all the copper atoms to separate the layers.

The modeling procedure is summarized in Fig. 2. The copper layer was set with a defined displacement, $d$ separated away from the epoxy plane. Then, the system was relaxed with successive energy minimization based on molecular statics (MS). The MS steps were carried out with the a cascade of steepest descent, ABNR, and quasi-Newton methods until the solution converged. ${ }^{28}$ The dynamics simulation was conducted at an equilibrium temperature of $298 \mathrm{~K}$ with velocity scale thermostat at an integration time step of 0.1 fs in a canonical ensemble (NVT). After the dynamics simulation, a final structure was output. The force acted on each epoxy atoms, in which $f_{i}$ was output for the traction and $F$ was the calculation. The procedure was repeated with increased set displacements in order to obtain the tractiondisplacement relationship.

The three influencing parameters which alter the interacting forces of the traction-displacement relations are highlighted inside the dotted box.

Traction-displacement relations are the functions that describe the traction (reaction forces) acting on one of the layers upon separation of an interface caused by the displacement of the other layer. In the epoxy-copper interface, the

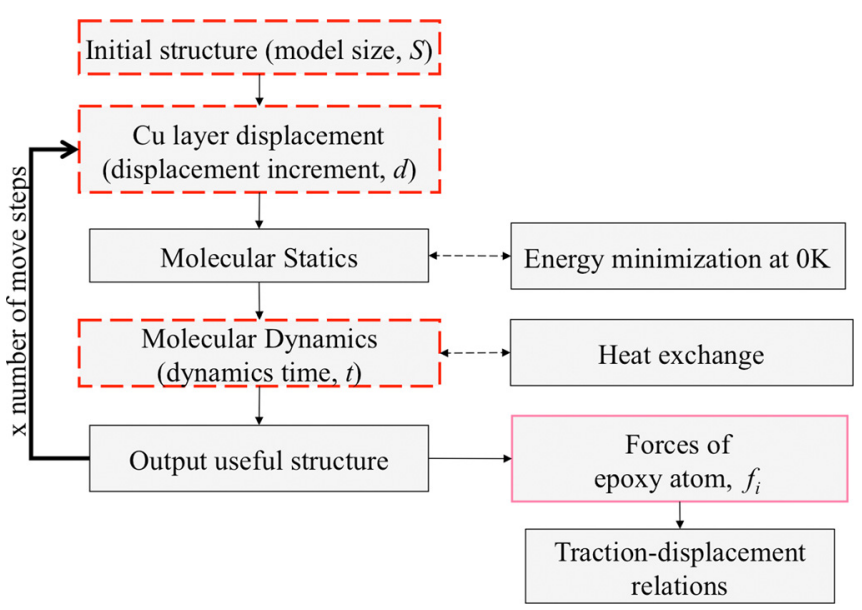

FIG. 2. Modeling procedure in obtaining the traction-displacement relations. The three influencing parameters which alter the interacting forces of the traction-displacement relations are highlighted inside the dotted box. 
a

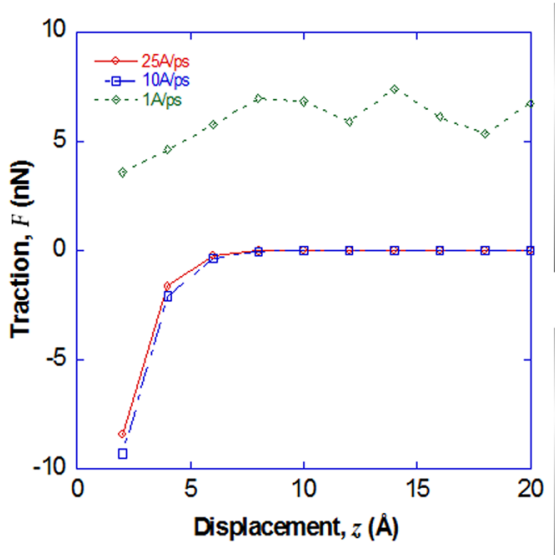

b

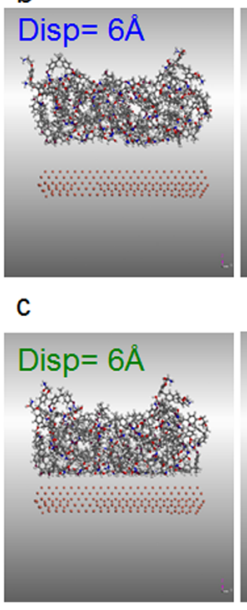

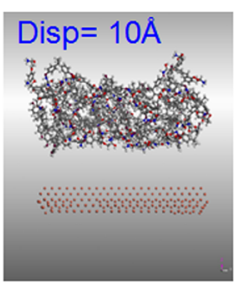

Disp $=20 \AA$
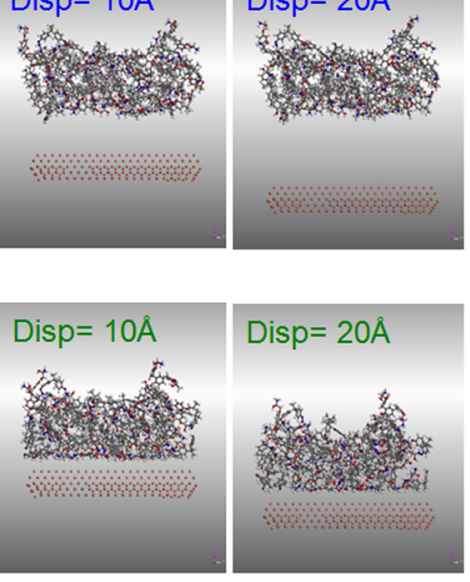

Disp $=20 \AA$

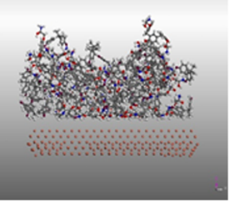

FIG. 3. (a) Traction-displacement functions in different displacement rates, $v$ : $25 \AA / p s, \quad 10 \AA / p s$, and $1 \AA / p s ; \quad$ Image sequence at (b) $10 \AA / p s$ showing the interface was separated; (c) $1 \AA / p s$ with no interface separation. traction acting on atoms in the epoxy layer in response to the copper layer displacement was calculated. The force, $f^{i}$, acting on an epoxy atom, $i$ can be determined from a pair potential, $V .{ }^{32-34}$

$$
f^{i}=-\sum_{i} \sum_{j} \frac{\partial V}{\partial r^{i j}} \frac{r_{\alpha}^{i j} r_{\beta}^{i j}}{\left|r_{\beta}^{i j}\right|},
$$

where $\alpha$ or $\beta$ corresponds to the $\mathrm{x}, \mathrm{y}, \mathrm{z}$ directions; $i$ and $j$ are atom indices; and $r$ is the position of the atom. The traction, $F$ was calculated as the summation of forces of these atoms in the epoxy layer (in z-direction) as shown in the Eq. (2),

$$
F=\sum_{i=1, n} f^{i}
$$

\section{INFLUENCING PARAMETERS FOR TRACTION-DISPLACEMENT RELATIONS}

Three influencing parameters (highlighted in Fig. 2), which can alter the interacting forces of the layers, were investigated: (1) time taken for the interaction of atoms in the dynamics step, $t$; (2) displacement increment of the copper layer which separates the interface in each step, $d$; and (3) model size that varies the molecular size of epoxy involves the interaction, $S$. The effect of time was conducted with a non-equilibrium MD simulation under a fixed displacement increment of $2 \AA$ for 10 steps. Different dynamics time was assigned as $t=0.08 \mathrm{ps}, 0.2 \mathrm{ps}$, and $2 \mathrm{ps}$ which accordingly varies the displacement rate as $v=25 \AA / \mathrm{ps}$, $10 \AA / p s$, and $1 \AA / p s$. To study the effect of displacement increment, the copper layer was separated from the epoxy in $-\mathrm{z}$ direction with $d=2 \AA, 1 \AA, 0.5 \AA, 0.4 \AA$, and $0.25 \AA$. In the model size investigation, three interfacial models of sizes, $S=14 \AA \times 14 \AA, 24 \AA \times 24 \AA$, and $28 \AA \times 56 \AA$ were evaluated.

Fig. 3(a) illustrates the traction-displacement functions calculated with the $25 \AA / p s, 10 \AA / p s$, and $1 \AA / p s$ displacement rate. At $10 \AA / \mathrm{ps}$ and $25 \AA / \mathrm{ps}$, the calculated tractions were independent on the displacement rate. However, at $1 \AA / \mathrm{ps}$, the result was not consistent with the higher displacement rate.
The shape of the functions can be explained by the image sequences taken at $10 \AA /$ ps and $1 \AA /$ ps in Figs. 3(b) and 3(c), respectively. In the $10 \AA / \mathrm{ps}$ case, the traction was about -9 $\mathrm{nN}$ at a displacement of $2 \AA$. The negative sign implies an attractive force exerted on the epoxy layer. The force then gradually reduced (became less negative) as the copper layer separated from the epoxy. As the displacement reached above $10 \AA$, the traction became zero, which meant no interacting forces between the two layers. This is consistent with the image sequence in Fig. 3(b), where a gradual separation of the two layers is clearly observed. In the $1 \AA /$ ps case, the traction varied at around $5 \mathrm{nN}$. With close observation of the image sequence in Fig. 3(c), the two layers were not separated while the copper layer was moved away. The traction did not vanish in this case. With no complete separation, the remaining interaction explains the non-zero forces. The result implies that it is essential to validate the displacement rate to be above a critical value to ensure the separation of the interface. The displacement rate was chosen as $10 \AA / p s$ in the following study.

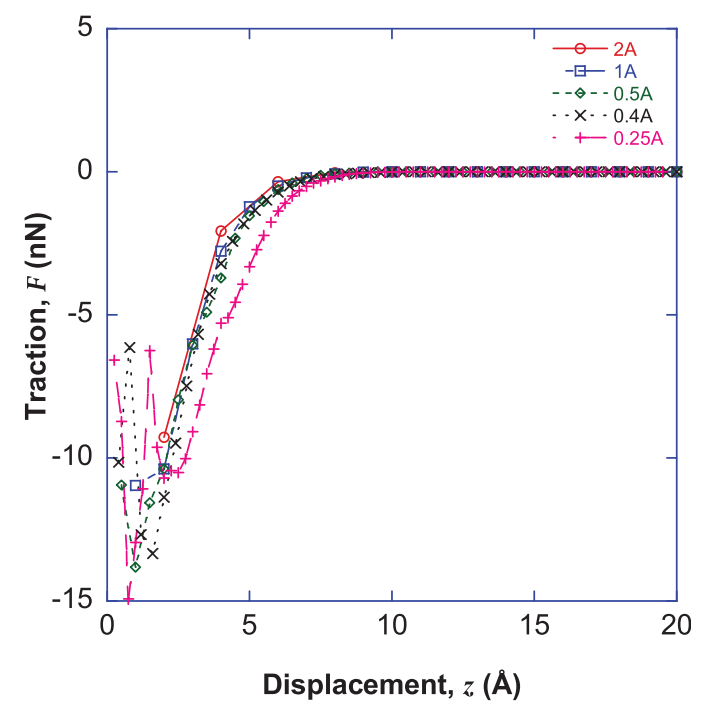

FIG. 4. Traction-displacement relations of the large $(28 \AA \times 56 \AA)$ model in different displacement increments. 


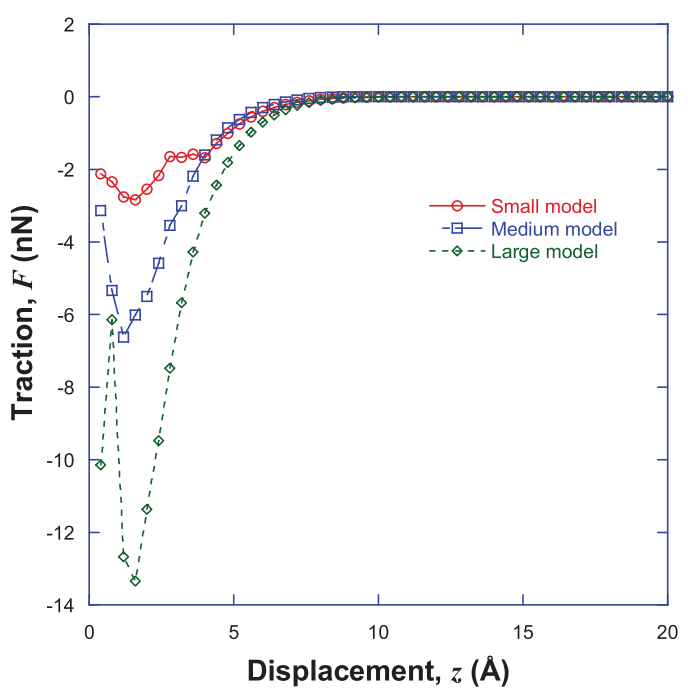

FIG. 5. Traction-displacement relations of the models in different model sizes.

Fig. 4 plots the traction-displacement functions in different displacement increments of the $28 \AA \times 56 \AA$ model at $10 \AA /$ ps. Similar trends were obtained from all the displacement increments. Fluctuation was shown with the $0.25 \AA$ data. The result revealed an energy instability as the dynamics time was set to be very small. In fact, a small displacement increment gives a better description of the interfacial separation when the layers are almost intact. However, with a short dynamics time of 0.025 ps for the $0.25 \AA$ displacement increment, the inadequate data points as induced by the instability lead to large fluctuations in the moving average.

The model size impact on the traction-displacement relations with a $0.4 \AA$ displacement increment at $10 \AA / \mathrm{ps}$ is illustrated in Fig. 5. Despite the similar trends in the traction-displacement relations, the traction values are different in the three models. Since the interaction involved different interfacial area, the interaction energy should be normalized with the model size as calculated in Sec. IV.

\section{INTERACTION ENERGY AND EQUILIBRIUM DISTANCE CALCULATION}

The interaction energy required to separate the interface, $\gamma_{T-d}$, can be determined from the change of the system energy upon separation. The interaction energy related to the traction-displacement function is given by Eq. (3),

TABLE I. Interaction energies of epoxy-copper system as obtained from different model sizes in different displacement increments.

\begin{tabular}{lccc}
\hline \hline & \multicolumn{3}{c}{ Interaction energy $\left(\mathrm{Jm}^{-2}\right)$} \\
\cline { 2 - 4 } $\begin{array}{l}\text { Displacement } \\
\text { increment, } d(\AA)\end{array}$ & $\begin{array}{c}\text { Small } \\
(14 \AA \times 14 \AA)\end{array}$ & $\begin{array}{c}\text { Medium } \\
(24 \AA \times 24 \AA)\end{array}$ & $\begin{array}{c}\text { Large } \\
(28 \AA \times 56 \AA)\end{array}$ \\
\hline 2 & -0.1948 & -0.1109 & -0.1482 \\
1 & -0.4160 & -0.2260 & -0.2034 \\
0.5 & -0.3738 & -0.2599 & -0.2394 \\
0.4 & -0.5131 & -0.3185 & -0.2342 \\
0.25 & -0.6020 & -0.2958 & -0.2800 \\
\hline \hline
\end{tabular}

$$
\gamma_{T-d}=-\frac{\partial U}{\partial A}=-\frac{\int_{z_{0}}^{z} F d z}{A-A_{0}},
$$

where $\partial U$ is the change in energy of the system when the copper layer displaces from $z_{0}$ to $z ; A$ is the area of the separated surface; $F$ is traction (reaction force) of the components in the epoxy layer upon separation; and $A_{0}$ is the separated area at the initial position $z_{0}$ which is assumed to be zero when interface is intact. The interaction energy, $\gamma_{T-d}$, can thus be calculated as the area under the traction-displacement functions divided by the interfacial area, $A$.

$$
\gamma_{T-d}=-\frac{\int_{z_{0}}^{z} F d z}{A} .
$$

In order to investigate the effect of the molecular size, three models with different size were simulated. The interaction energies required to separate the interface, $\gamma_{T-d}$ were calculated by Eq. (4). Table I summarizes the interfacial energies calculated from the traction-displacement functions in different model sizes.

Fig. 6 illustrates the plot of the interaction energies, $\gamma_{T-d}$ against displacement increments in different model sizes. The traction-displacement functions obtained with a smaller displacement increment gives more detailed description for the separation at intimate contact and normally can give better parameters estimation. However, it is impossible to reduce the displacement increment indefinitely. In order to evaluate the interaction energies, $\gamma$, at intimate contact, a linear regression fitting with extrapolation is used to extract the value from the zero displacement. The interaction energies of the small, medium and large models are $-0.581 \mathrm{Jm}^{-2}$, $-0.334 \mathrm{Jm}^{-2}$, and $-0.276 \mathrm{Jm}^{-2}$, respectively.

Fig. 7 plots the extrapolated interfacial energies, $\gamma$ against the model sizes, $S$. As indicated by the trend line, the

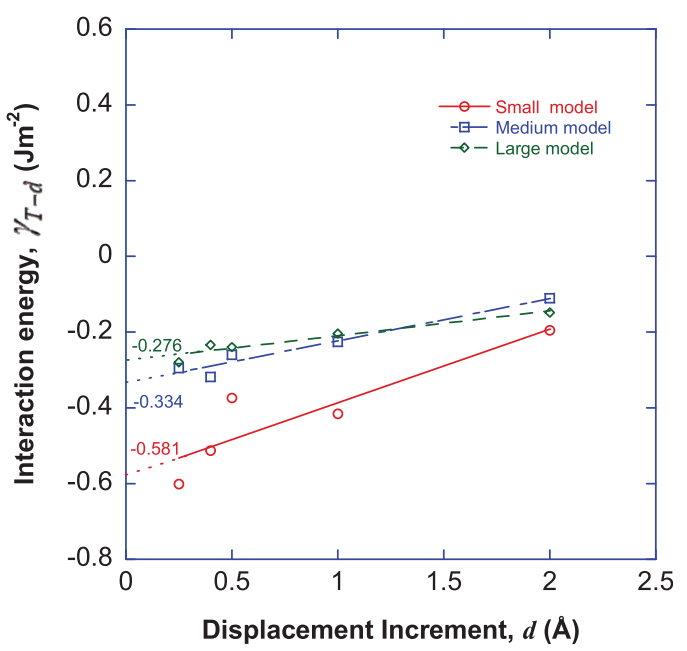

FIG. 6. The interaction energies in different model sizes as obtained by the extrapolating the fitting line in the interaction energies-displacement increments plot. 


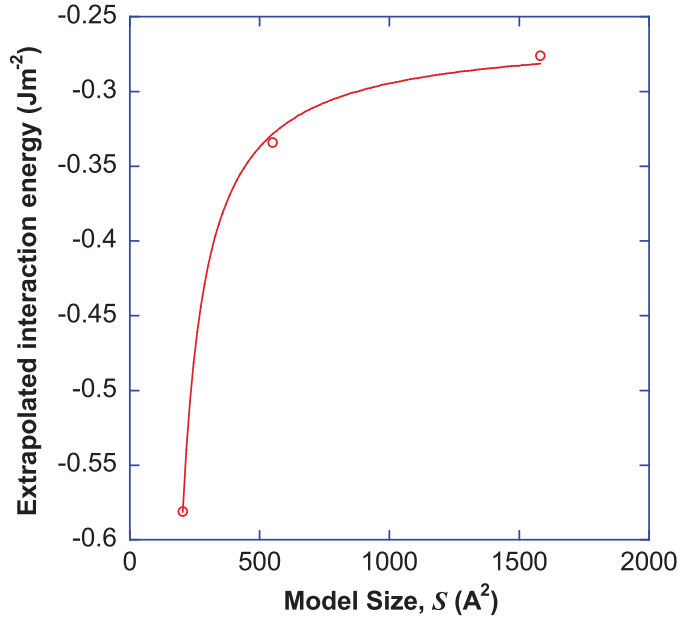

FIG. 7. Model size verification-a flattened trend line is achieved with the large model $(28 \AA \times 56 \AA)$.

interaction energy significantly increases with the model size from $14 \AA \times 14 \AA$ to $24 \AA \times 24 \AA$. With model size beyond $24 \AA \times 24 \AA$, the increase of the interaction energy plateaus out with larger molecules. The interaction energy is expected to be constant with further increase of the model size beyond $28 \AA \times 56 \AA$. As a result, the interaction energy of about $-0.276 \mathrm{Jm}^{-2}$ is considered for the material system beyond nano-size.

To benchmark the result, the interaction energy as discerned from the traction-displacement functions is compared with the thermodynamics work of adhesion as derived from contact angle experiments. ${ }^{35}$ Table II compares the interaction energy calculated from (1) the traction-displacement functions; and (2) thermodynamics work of adhesion. The values are in close agreement.

The equilibrium distance between the interfacial materials at a minimum energy, $r_{e}$ can be determined from the traction-displacement plots. By fitting the plots with the derivative of the Morse potential (see Eq. (A2) in Appendix), the equilibrium distance is determined as a traction minimum. Fig. 8 shows the traction-displacement plots with a set displacement increment of $0.4 \AA$. The plot was fitted with the derivative of a Morse potential. The equilibrium distance deduced from the minimum point is $1.2 \AA$.

\section{COARSE GRAINED MODEL PARAMETERS}

Table III summarizes the interfacial energies (as converted to $\mathrm{kcal} \mathrm{mol}^{-1}$ ) and the equilibrium distances in differ-

TABLE II. Interaction energy of epoxy-copper system as obtained from different models.

\begin{tabular}{lcc}
\hline \hline Method & $\begin{array}{c}\text { Interaction } \\
\text { energy }\left(\mathrm{Jm}^{-2}\right)\end{array}$ & Function \\
\hline Traction-displacement relations & -0.276 & $\gamma_{T-d}=-\frac{\int_{z_{0}}^{z} F d z}{A}$, \\
Thermodynamics work of adhesion & $-0.26^{\mathrm{a}}$ & $W_{A}=\gamma_{a}+\gamma_{s}-\gamma_{a s}$ \\
\hline \hline
\end{tabular}

${ }^{\mathrm{a}}$ Negative means energy is required to separate the interface.

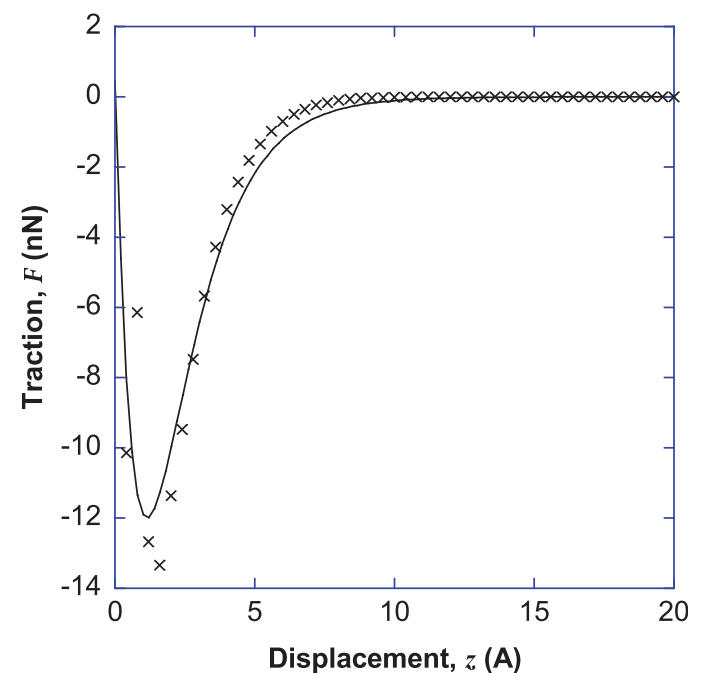

FIG. 8. Curve fitting of the traction-displacement function $(d=0.4 \AA)$ with the derivative of Morse potential (Eq. (A2)), the equilibrium distance which determined as the minimum point is $1.2 \AA$.

ent displacement increments for the model with size of $28 \AA \times 56 \AA$.

To estimate the parametric values at the state of intimate contact, plots of the equilibrium distance and the interaction energy against the displacement increment are conducted as illustrated in Fig. 9. The values are obtained from linear regression fitting with extrapolation to displacement increment at zero. The deduced interaction energy and equilibrium distance from this method are $-62.8 \mathrm{kcal} \mathrm{mol}^{-1}$ and $2.2 \AA$, respectively. The results were closed match with value of $-64 \mathrm{kcal} \mathrm{mol}^{-1}$ and $2.1 \AA$ reported by Iwamoto ${ }^{21}$ who conducted a single point energy calculation of an epoxycopper system.

\section{DISCUSSION}

The methodology to determine the coarse grained parameters of the separation process of an epoxy-metal interface is established using molecular dynamics simulation. The investigations reveal that there are several model settings that have to be carefully controlled. The recommendations on modeling are summarized in the following:

(1) The control of transient time allowed for the atomic interaction has been found critical. The dynamics simulation was intended to eliminate the artificial bonds stretching in the molecules. Typically, a longer simulation time step is set to collect more data points for a time

TABLE III. The interaction energy and equilibrium distance of epoxycopper system as obtained from models with different increments.

\begin{tabular}{lcc}
\hline \hline $\begin{array}{l}\text { Displacement } \\
\text { increment, } d(\AA)\end{array}$ & $\begin{array}{c}\text { Interaction energy, } \\
\gamma\left(\mathrm{kcal} \mathrm{mol}^{-1}\right)\end{array}$ & $\begin{array}{r}\text { Equilibrium } \\
\text { distance, } r_{e}(\AA)\end{array}$ \\
\hline 2 & -33.7 & - \\
1 & -46.3 & - \\
0.5 & -54.5 & 1.0 \\
0.4 & -53.3 & 1.2 \\
0.25 & -63.7 & 1.6 \\
\hline \hline
\end{tabular}



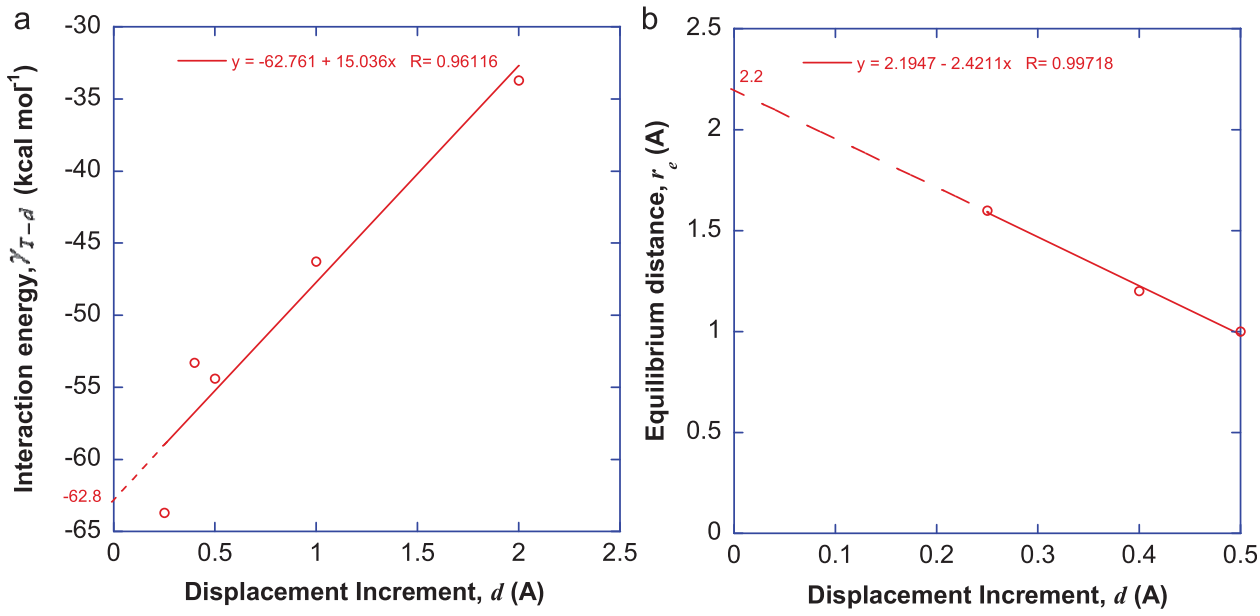

FIG. 9. Determination of the coarse grained parameters. (a) the interaction energy and (b) equilibrium distance by extrapolation to the $y$-axis. averaging result that eliminates error caused by numerical randomness. However, for the bi-material system that undergoes separation, too long a time step, may allow the attraction of the two materials and hampers the system separation as illustrated from our results.

(2) The apparent interaction energy can be size dependent when the molecules are small. Based on the results and the benchmarking with other study, it is advised that the interfacial area of the model to be above $28 \AA \times 56 \AA$ when the interaction energy is representing the material with size above nano-scale.

(3) The coarse grained parameters at the intimate contact state were determined from the corresponding parameters-displacement increment function $\left(\gamma-d\right.$ or $\left.r_{e^{-}} d\right)$ using linear regression fitting with extrapolation to zero displacement increment. The proposed approach overcomes the difficulties of simulating the traction at infinitesimal small separation. The calculated results are well match with the values reported from other studies.

The study demonstrates a validated modeling procedure in studying the separation process of an epoxy-metal system having no chemical bonding linkages at the interface. It is anticipated that the procedure can further be adopted to study the interfacial separation of a system involves chemical bonding interactions. Given the bond breaking criteria are available, the proposed approach can be extended to investigate other irreversible failure phenomenon observed during interfacial separation.

\section{CONCLUSIONS}

In summary, a non-equilibrium MD model to calculate the traction-displacement relations of a separation process between epoxy and copper is established. The study illustrates that the control of the time step in the molecular dynamics models is important. Selection of a displacement rate above critical is essential to ensure a proper separation simulation. This paper demonstrates a methodology in obtaining the coarse grained parameters from the tractiondisplacement functions of an interfacial separation. The method is potentially capable to study interfacial separation involves chemical bonds along the interface given that the bond breaking criteria is available. The coarse grained pa- rameters can be beneficial for the understanding of the interfacial separation observed in macroscopic point of view.

\section{ACKNOWLEDGMENTS}

The work is financed by the European Commission under project NanoInterface (NMP-2008-214371).

\section{APPENDIX: SUPPORTING INFORMATION}

The relaxation steps in obtaining the initial structure of the interfacial model, the equation of the Morse potential and its derivative are presented.

\section{Equation (A1) Morse potential}

The functional form of the Morse potential is given as:

$$
U(r)=D\left(1-e^{-2 a\left(r-r_{e}\right)}\right)^{2} .
$$

Here $r$ is the distance between the atoms, $r_{e}$ is the equilibrium bond distance, $D$ is the well depth (defined relative to the dissociated atoms), and $a$ controls the width of the potential.

\section{Equation (A2) derivative of the Morse potential}

The force, $F$ involves in the separation of the atoms can be calculated from the derivative of the potential energy against the separation distance, $r$ as shown in the Eq. (A2)

$$
F=\frac{\partial U}{\partial r}=D e^{-2 a r}\left(2 a e^{a r+a r_{e}}-2 a e^{2 a r_{e}}\right) .
$$

TABLE IV. Relaxation procedures for the interfacial model.

\begin{tabular}{lc}
\hline \hline Step & MS/MD steps \\
\hline 1 & Energy minimization at $0 \mathrm{~K}$ \\
2 & NVT at $298 \mathrm{~K}, 5 \mathrm{ps}, 0.1$ fs per step \\
3 & Annealing at $298 \mathrm{~K}$ to $500 \mathrm{~K}, 10$ cycles ( 5 heat ramps per cycle) \\
4 & for $10 \mathrm{ps}, 0.1 \mathrm{fs}$ per step \\
\hline \hline
\end{tabular}


${ }^{1}$ A. J. Kinloch, Adhesion and Adhesives: Science and Technology (Springer, London, 1987).

${ }^{2}$ K. Mittal, Pure Appl. Chem. 52, 5 (1980).

${ }^{3}$ M. J. Hinton, A. Kaddour, and P. D. Soden, Failure Criteria in Fibre Reinforced Polymer Composites: The World-Wide Failure Exercise (Elsevier Science, San Diego, 2004).

${ }^{4} \mathrm{M}$. Pecht, Integrated Circuit, Hybrid, and Multichip Module Package Design Guidelines: A Focus on Reliability (Wiley-Interscience, New York, 1994).

${ }^{5}$ H. M. Duong, N. Yamamoto, K. Bui, D. V. Papavassiliou, S. Maruyama, and B. L. Wardle, J. Phys. Chem. C 114(19), 8851 (2010).

${ }^{6}$ J. Lin, H. Zhang, Z. Chen, and Y. Zheng, ACS nano 4(9), 5421 (2010).

${ }^{7}$ D. L. Patrick, J. F. Flanagan IV, P. Kohl, and R. M. Lynden-Bell, J. Am. Chem. Soc. 125(22), 6762 (2003).

${ }^{8}$ J. D. Schall, G. Gao, and J. A. Harrison, J. Phys. Chem. C 114(12), 5321 (2009).

${ }^{9}$ Q. Zheng, Q. Xue, K. Yan, L. Hao, Q. Li, and X. Gao, J. Phys. Chem. C 111(12), 4628 (2007).

${ }^{10}$ K. Gall, M. Horstemeyer, M. Van Schilfgaarde, and M. Baskes, J. Mech. Phys. Solids 48(10), 2183 (2000).

${ }^{11}$ C. A. Schuh and A. C. Lund, Nature Mater. 2(7), 449 (2003).

${ }^{12}$ A. Cao and Y. Wei, Phys. Rev. B 74(21), 214108 (2006).

${ }^{13}$ H. Lee and R. G. Larson, J. Phys. Chem. B 112(26), 7778 (2008).

${ }^{14}$ S. Li, X. Zhang, and W. Wang, J. Phys. Chem. B 113(43), 14431 (2009).

${ }^{15}$ J. McCarty, I. Lyubimov, and M. Guenza, J. Phys. Chem. B 113(35), 11876 (2009).

${ }^{16}$ T. R. Prytkova, I. Eryazici, B. Stepp, S. B. Nguyen, and G. C. Schatz, J. Phys. Chem. B 114(8), 2627 (2010).

${ }^{17}$ G. Rossi, I. Giannakopoulos, L. Monticelli, N. K. J. Rostedt, S. R. Puisto, C. Lowe, A. C. Taylor, I. Vattulainen, and T. Ala-Nissila, Macromolecules 44(15), 6198 (2011).

${ }^{18}$ K. Liao and S. Li, Appl. Phys. Lett. 79(25), 4225 (2001).
${ }^{19}$ S. Namilae and N. Chandra, J. Eng. Mater. Technol. 127, 222 (2005).

${ }^{20}$ T. Semoto, Y. Tsuji, and K. Yoshizawa, J. Phys. Chem. C 115(23), 11707 (2011).

${ }^{21}$ N. Iwamoto, Microelectron. Reliab. 1035 (2011).

${ }^{22}$ R. DeVane, M. L. Klein, C. Chiu, S. O. Nielsen, W. Shinoda, and P. B. Moore, J. Phys. Chem. B 114(19), 6386 (2010).

${ }^{23}$ J. C. Shelley, M. Y. Shelley, R. C. Reeder, S. Bandyopadhyay, and M. L. Klein, J. Phys. Chem. B 105(19), 4464 (2001).

${ }^{24}$ S. Yang, F. Gao, and J. Qu, in Proceedings of the 60th Electronic Components and Technology Conference (ECTC), Las Vegas, NV, USA, 2010.

${ }^{25}$ A. R. Leach, Molecular Modelling: Principles and Applications (AddisonWesley Longman Ltd., 2001).

${ }^{26}$ O. Holck, E. Dermitzaki, B. Wunderle, J. Bauer, B. Michel, and H. Reichl, in Proceedings of the 60th Electronic Components and Technology Conference (ECTC) (Las Vegas, NV, USA, 2010).

${ }^{27}$ S. L. Mielke, T. Belytschko, and G. C. Schatz, Annu. Rev. Phys. Chem. 58, 185 (2007).

${ }^{28}$ Accelrys. Materials studio release notes, release 5.0, (2009).

${ }^{29}$ R. Toth, M. Ferrone, S. Miertus, E. Chiellini, M. Fermeglia, and S. Pricl, Biomacromol. 7(6), 1714 (2006).

${ }^{30}$ B. Prathab, V. Subramanian, and T. Aminabhavi, Polymer 48(1), 409 (2007).

${ }^{31}$ S. Kisin, J. B. Vukic, P. G. T. van der Varst, Gijsbertus de With, and C. E. Koning, Chem. Mater. 19(4), 903 (2007).

${ }^{32}$ N. Chandra, S. Namilae, and C. Shet, Phys. Rev. B 69(9), 94101 (2004).

${ }^{33}$ S. Namilae and N. Chandra, J. Eng. Mater. Technol. 127, 222 (2005).

${ }^{34}$ S. Namilae and N. Chandra, ASME J. Eng. Mater. Technol. 127, 222 (2005).

${ }^{35} \mathrm{~T}$. Ferguson and $\mathrm{J}$. Qu, in Micro-and Opto-Electronic Materials and Structures: Physics, Mechanics, Design, Reliability, Packaging, edited by E. Suhir, Y. C. Lee, and C. P. Wong (Springer, New York, 2007), p. 431. 